COMMUNICATIONS IN

NUMBER THEORY AND PHYSICS

Volume 7, Number 4, 671-687, 2013

\title{
Integrality of relative BPS state counts of toric del Pezzo surfaces
}

\author{
Michel van Garrel, Tony W.H. Wong and Guergui Zaimi
}

\begin{abstract}
Relative Bogomolny-Prasad-Sommerfield (BPS) state counts for log Calabi-Yau surface pairs were introduced by Gross-Pandharipande-Siebert in [4] and conjectured by the authors to be integers. For toric del Pezzo surfaces, we provide an arithmetic proof of this conjecture, by relating these invariants to the local BPS state counts of the surfaces. The latter were shown to be integers by Peng in [15]; and more generally for toric Calabi-Yau three-folds by Konishi in [8].
\end{abstract}

\section{Introduction}

\subsection{Local BPS state counts}

For Calabi-Yau three-folds, BPS invariants were defined by GopakumarVafa in $[5,6]$ using a $M$-theory construction. Their definition and the related conjectures were extended to all three-folds by Pandharipande in $[13,14]$. Presently, genus 0 invariants are considered, also called BPS state counts. For the local Calabi-Yau geometries relevant below, the terminology local BPS state counts is used. Let $S$ be a smooth del Pezzo surface and denote by $E$ a smooth effective anticanonical divisor on it, that is, an elliptic curve. Denote furthermore by $K_{S}$ the non-compact local Calabi-Yau three-fold given as the total space of the canonical bundle $\mathcal{O}_{S}(-E)$ on $S$. For a curve class $\beta \in H_{2}(S, \mathbb{Z})$, denote by $n_{\beta}$ the local BPS state count in class $\beta$, whose definition we state below. From a physics point of view, $n_{\beta}$ counts $D$-branes supported on genus 0 curves of class $\beta$. This definition does not rest on rigorous mathematical foundations, so alternative definitions are used. Perhaps the most common one, the one we follow in this paper, aims at extracting multiple cover contributions from Gromov-Witten invariants and is as follows. Denote by $\bar{M}_{0,0}(S, \beta)$, resp. by $\bar{M}_{0,1}(S, \beta)$, the moduli stack of stable maps $f: C \rightarrow S$ from genus 0 curves with no, resp. one, marked point to $S$ such that $f_{*}([C])=\beta$. Denote moreover by $\pi: \bar{M}_{0,1}(S, \beta) \rightarrow \bar{M}_{0,0}(S, \beta)$ the forgetful morphism and by ev : $\bar{M}_{0,1}(S, \beta) \rightarrow S$ the evaluation map. This 
determines the obstruction bundle $R^{1} \pi_{*} \mathrm{ev}^{*} K_{S}$ whose fiber over a stable map $f: C \rightarrow S$ is $H^{1}\left(C, f^{*} K_{S}\right)$. Then $I_{K_{S}}(\beta)$, the genus 0 local Gromov-Witten invariant of degree $\beta$ of $S$, is defined as the integral of the Euler class of $R^{1} \pi_{*} \mathrm{ev}^{*} K_{S}$ against the virtual fundamental class of $\bar{M}_{0,0}(S, \beta)$, i.e.,

$$
I_{K_{S}}(\beta):=\int_{\left[\bar{M}_{0,0}(S, \beta)\right]^{\text {vir }}} \mathrm{e}\left(R^{1} \pi_{*} \mathrm{ev}^{*} K_{S}\right) \in \mathbb{Q} .
$$

The definition of the associated BPS state counts is modeled on the following ideal (rarely satisfied) situation: suppose that $K_{S}$ only contained a finite number of genus 0 degree $\beta$ curves, and that all these curves were rigidly embedded in $K_{S}$, i.e., with normal bundle isomorphic to $\mathcal{O}(-1) \oplus \mathcal{O}(-1)$. Then $n_{\beta}$ should be the number of such curves. Let $\tilde{C} \subset K_{S}$ be such a rigid rational curve of degree $\beta$. According to the Aspinwall-Morrison formula proven by Manin in [10], degree $k$ stable maps

$$
C \rightarrow \operatorname{Tot}\left(\mathcal{O}_{\tilde{C}}(-1) \oplus \mathcal{O}_{\tilde{C}}(-1)\right)
$$

contribute a factor of $\frac{1}{k^{3}}$ to the Gromov-Witten invariant $I_{K_{S}}(k \beta)$. For $k \in$ $\mathbb{N}$, we write $k \mid \beta$ to mean that there is $\beta^{\prime} \in H_{2}\left(K_{S}, \mathbb{Z}\right)$ such that $k \beta^{\prime}=\beta$. In this ideal situation then, the following equality would hold:

$$
I_{K_{S}}(\beta)=\sum_{k \mid \beta} \frac{1}{k^{3}} n_{\beta / k}
$$

In general, the described geometric conditions are not satisfied, and so the $n_{\beta}$ do not count curves in class $\beta .^{1}$ They can nonetheless be defined via Equation (1.1), or alternatively, via generating functions as follows.

Definition 1 (Stated as a formula by Gopakumar-Vafa in $[5,6]$; stated as a definition by Bryan-Pandharipande in [1]). Assume $\beta$ to be primitive. Then the local BPS state counts $n_{d \beta}$, for $d \geq 1$, are defined as rational numbers via the formula

$$
\sum_{l=1}^{\infty} I_{K_{S}}(l \beta) q^{l}=\sum_{d=1}^{\infty} n_{d \beta} \sum_{k=1}^{\infty} \frac{1}{k^{3}} q^{d k} .
$$

Conjecture 2. (Attributed to Gopakumar-Vafa; stated in [1] by Bryan-Pandharipande) For all curve classes $\beta \in H_{2}(S, \mathbb{Z})$,

$$
n_{\beta} \in \mathbb{Z}
$$

\footnotetext{
${ }^{1}$ It is believed though that a (non-algebraic) deformation of $K_{S}$ exhibits these conditions.
} 
Conjecture 2 was proven by Peng in [15] in the case of toric del Pezzo surfaces, which are the del Pezzo surfaces of degree $\geq 6$. More generally, a proof for toric Calabi-Yau three-folds was given by Konishi in [8].

\subsection{Relative BPS state counts}

The definitions and conjectures relating to relative BPS state counts mirror the discussion of the previous section. These invariants were introduced by Gross-Pandharipande-Siebert in [4]. Whereas the previous section is concerned with local Calabi-Yau three-folds, this one deals with open CalabiYau surfaces, which are examples of log Calabi-Yau surfaces.

Definition 3 (See [4]). Let $S$ be a smooth surface and let $D \subset S$ a smooth divisor. Let furthermore $\gamma \in H_{2}(S, \mathbb{Z})$ be non-zero. The pair $(S, D)$ is called $\log$ Calabi-Yau with respect to $\gamma$ if

$$
D \cdot \gamma=c_{1}(S) \cdot \gamma
$$

If Equation (1.3) holds for all $\gamma \in H_{2}(S, \mathbb{Z})$, which is the situation considered below, we abbreviate and say that $(S, D)$ is a $\log$ Calabi-Yau surface pair. Sometimes the divisor $D$ is excluded from the notation.

The discussion in [4] is concerned with any log Calabi-Yau surface pair. For our purposes, we restrict to del Pezzo surfaces with a smooth anticanonical divisor. We mention in Section 1.5 below an integrality result by Reineke in [18], which concerns relative BPS state counts associated to blow ups of the projective plane relative to the toric divisor. ${ }^{2}$

As in the previous section, let $S$ be a del Pezzo surface and denote by $E$ a smooth effective anticanonical divisor on it. Then the pair $(S, E)$ is log Calabi-Yau. This is the open Calabi-Yau geometry considered in this section. This terminology is justified by the fact that the canonical bundle of $S$ is trivial away from $E$. Let $\beta \in H_{2}(S, \mathbb{Z})$ be the class of a curve and set $w=E \cdot \beta$. Note that the moduli stack of genus 0 curves in $S$ is of virtual dimension $w-1$. A generic curve representing $\beta$ would meet $E$ in $w$ points of simple tangency. Instead, we can impose that the curve meets

\footnotetext{
${ }^{2}$ The precise geometry is more elaborate. In particular, in order to obtain a smooth divisor, the singular points of the toric divisor are removed. The authors in [4] prove that (contrary to expectation) invariants can be defined for this open geometry.
} 
$E$ in fewer points with higher tangencies, cutting down the virtual dimension. Considering the maximal case, denote by $\bar{M}(S / E, w)$ the moduli stack which roughly parametrizes genus 0 relative stable maps $f: C \rightarrow S$ representing $\beta$ and such that the image of $C$ meets $E$ in one point of tangency $w$. Then $\bar{M}(S / E, w)$ is of virtual dimension 0 and the degree of its virtual fundamental class

$$
N_{S}[w]:=\int_{[\bar{M}(S / E, w)]^{\mathrm{vir}}} 1 \in \mathbb{Q}
$$

is called the genus 0 relative Gromov-Witten invariant of degree $\beta$ and maximal tangency of $(S, E)$. Denote by $\iota: P \rightarrow S$ a rigid element of $\bar{M}(S / E, w)$. For $k \geq 1$, denote by $M_{P}[k]$ the contribution of $k$-fold multiple covers of $P$ to $N_{S}[k w]$ (see [4] for precise definitions).

Proposition 4 (Proposition 6.1 in [4]).

$$
M_{P}[k]=\frac{1}{k^{2}}\left(\begin{array}{c}
k(w-1)-1 \\
k-1
\end{array}\right) .
$$

Consequently:

Definition 5 (Paragraph 6.3 in [4]). For $d \geq 1$, the relative BPS state counts $n_{S}[d w] \in \mathbb{Q}$ are defined by means of the equality

$$
\sum_{l=1}^{\infty} N_{S}[l w] q^{l}=\sum_{d=1}^{\infty} n_{S}[d w] \sum_{k=1}^{\infty} \frac{1}{k^{2}}\left(\begin{array}{c}
k(d w-1)-1 \\
k-1
\end{array}\right) q^{d k}
$$

Conjecture 6 (Conjecture 6.2 in [4]). Let $\beta \in H_{2}(S, \mathbb{Z})$ be an effective curve class and set $w=\beta \cdot E$. Then, for all $d \geq 1$

$$
n_{S}[d w] \in \mathbb{Z}
$$

\subsection{Main result}

Our main result is based on the following theorem, which was proved for $\mathbb{P}^{2}$ by Gathmann in [3]. A proof for all del Pezzo surfaces was announced by Graber-Hassett.

Theorem 7 (Gathmann for $\mathbb{P}^{2}$ in [3], for general $S$ announced by Graber-Hassett). Let $S$ be a del Pezzo surface and denote by $E$ a smooth effective anticanonical divisor on it. Let $\beta \in H_{2}(S, \mathbb{Z})$ be an effective 
Integrality of relative BPS state counts of toric del Pezzo surfaces 675 curve class and set $w=\beta \cdot E$. Then the following identity of Gromov-Witten invariants holds:

$$
N_{S}[w]=(-1)^{w+1} w I_{K_{S}}(\beta)
$$

In the present paper, we prove the following theorem:

Theorem 8. Let $\beta \in H_{2}(S, \mathbb{Z})$ be an effective non-zero primitive curve class. Consider two sequences of rational numbers

$$
\left\{N_{S}[d w]\right\}_{d \geq 1} \quad \text { and } \quad\left\{I_{K_{S}}(d \beta)\right\}_{d \geq 1}
$$

and assume that they are related, for all $d \geq 1$, via (cf. Equation (1.5))

$$
N_{S}[d w]=(-1)^{d w+1} d w I_{K_{S}}(d \beta) .
$$

Define two sequences of rational numbers

$$
\left\{n_{S}[d w]\right\}_{d \geq 1} \quad \text { and } \quad\left\{n_{d \beta}\right\}_{d \geq 1}
$$

by means of Equations (1.2) and (1.4). Then

$$
n_{S}[d w] \in \mathbb{Z} \quad \text { for all } d \geq 1
$$

if and only if

$$
d w \cdot n_{d \beta} \in \mathbb{Z} \text { for all } d \geq 1
$$

An immediate consequence is as follows:

Corollary 9. Conjecture 2 for $K_{S}$ implies Conjecture 6 for $(S, E)$.

Per the integrality result of Peng in [15] or of Konishi in [8] then:

Corollary 10. Conjecture 6 holds for toric del Pezzo surfaces.

Our proof of Theorem 8 employs some of the same methods that Peng used in his proof of Conjecture 2 for toric del Pezzo surfaces, cf. [15]. Namely, 
we rely on congruence relations between binomial coefficients. In particular, Lemma 13 below was stated in [15].

\subsection{Relationship to Takahashi's work on log mirror symmetry}

It follows from Theorem 7 that the relative BPS state counts are related to the local BPS state counts (see Lemma 12 below for the precise relationship). The local invariants are calculated via mirror symmetry (see ChiangKlemm-Yau-Zaslow in [2]) and thus, it is expected that the relative BPS state counts are directly computed via mirror symmetry as well. It is not clear what the $B$-model is though and there is at present no physical interpretation for these relative Gromov-Witten invariants. A mirror symmetry conjecture in this sense was formulated and explored by Takahashi in [19] for the projective plane. Let us note though that Takahashi considers an alternative enumerative version of relative BPS state counts.

Takahashi develops logarithmic mirror symmetry for $\mathbb{P}^{2}$ relative to an elliptic curve $E$, and considers the following $A$-model invariants. Let $d \geq 1$. A degree $d$ curve in $\mathbb{P}^{2}$ will meet $E$ in a $3 d$-torsion point. Choose a group structure on $E$ such that the zero element $0 \in E$ is a flex point. Choose $P \in E$ a point of order $3 d$ for the chosen group structure. Then $m_{d}$ is defined as the number of rational degree $d$ curves in $\mathbb{P}^{2}$ meeting $E$ only at $P$ in only one branch. The relative BPS state counts $n_{\mathbb{P}^{2}}[3 d]$ are a virtual extension of $m_{d}$ in the sense that the rational curves virtually counted by $n_{\mathbb{P}^{2}}[3 d]$ are allowed to meet $E$ in any $3 d$-torsion point, not just at $P$. The attribute virtual is justified since the $n_{\mathbb{P}^{2}}[3 d]$ arise from Gromov-Witten invariants. Based on his calculations and on the work by Gathmann in [3], Takahashi conjectures that the $m_{d}$ are related to the local BPS state counts $n_{d}$ of $\mathbb{P}^{2}$ as follows.

\section{Conjecture 11 (Takahashi in [19]).}

$$
3 d m_{d}=(-1)^{d+1} n_{d} .
$$

The above conjecture provides an enumerative interpretation of the invariants $n_{d}$. We prove a result analogous to Conjecture 11 in Lemma 12 below. Namely, that lemma provides a linear relationship between the sets of invariants $n_{\mathbb{P}^{2}}[3 d]$ and $n_{d}$. It follows that the relative BPS state counts are calculated from the periods of the mirror family. This is more generally true for any del Pezzo surface, since Lemma 12 holds in that setting. 


\subsection{Integrality of relative BPS state counts of another geometry}

The relative BPS state counts of Definition 5 are defined in [4] in the more general setting of log Calabi-Yau surface pairs (see Definition 3 above), and the integrality Conjecture 6 is stated in that generality. An example treated in detail throughout [4] is that of the pairs consisting of blow ups of weighted projective planes and their toric divisors. ${ }^{3}$ Consider $(a, b) \in \mathbb{N}^{2}$ determining a weighted projective plane via the action

$$
t \cdot(x, y \cdot z)=\left(t^{a} x, t^{b} y, t z\right)
$$

where $t \in \mathbb{C}^{\times}$. For $k \in \mathbb{N}$, let moreover

$$
\mathbf{P}=\left(\mathbf{P}_{a}, \mathbf{P}_{b}\right)
$$

consists of two ordered partitions

$$
\mathbf{P}_{a}=p_{1}+\cdots+p_{l_{a}} \quad \text { and } \quad \mathbf{P}_{b}=p_{1}^{\prime}+\cdots+p_{l_{b}}^{\prime}
$$

of sizes $a k$, resp. $b k$. Then the authors consider relative Gromov-Witten invariants, denoted by

$$
N_{a, b}[\mathbf{P}] \in \mathbb{Q}
$$

which, roughly speaking, count genus 0 maps with prescribed intersection multiplicities along the toric divisors. ${ }^{4}$ Note that $k$ is implicit in the notation. In [18], Reineke-Weist prove that the data of the Gromov-Witten invariants $N_{a, b}[\mathbf{P}]$ are equivalent to the data given by the Euler characteristic of moduli spaces of quiver representations. Using this correspondence, the authors prove in [18, Corollary 11.4] a variant of Conjecture 6 above. They prove that BPS state counts extracted from the projective space invariants $N_{1,1}[\mathbf{P}]$, for appropriate partitions $\mathbf{P}$, are integers. The above Corollary 10 can be thought of as an analogue of that result in the case of a smooth non-toric anticanonical divisor.

\footnotetext{
${ }^{3}$ More precisely, the singular points of the toric divisors are removed since the divisors of log Calabi-Yau surface pairs are required to be smooth. The authors prove that the standard techniques yield well-defined invariants.

${ }^{4}$ See [4] for the exact statements concerning the geometry, the intersection multiplicities and the well definedness of the invariants.
} 


\subsection{Relationship to the integrality of Donaldson-Thomas-type invariants}

Gromov-Witten invariants are defined via intersection theory on the stable map compactification. ${ }^{5}$ Donaldson-Thomas invariants are a closely related way of counting curves, corresponding to the Hilbert space compactification. ${ }^{6}$ A generalization of Donaldson-Thomas invariants was proposed in [9] by Kontsevich-Soibelman and in [7] by Joyce-Song. A special type of such invariants is considered by Reineke in [17]. Fix $m \geq 1$. In [17, Definition 3.3], the author considers the following Donaldson-Thomas type invariants. Let $n \geq 0$. Then $\mathrm{DT}_{n}^{(m)}$ encodes the Euler characteristic of some specific non-commutative Hilbert schemes associated to the $m$-loop quiver. More precisely, consider the free algebra on $m$ generators $F^{(m)}:=\mathbb{C}\left\langle x_{1}, \ldots, x_{m}\right\rangle$. Consider, moreover, the non-commutative Hilbert scheme $\mathrm{Hilb}_{n}^{(m)}$ parametrizing left ideals $I$ in $F^{(m)}$ of codimension $n$, i.e., such that $\operatorname{dim}_{\mathbb{C}} F^{(m)} / I=n$. Consider furthermore the generating function of Euler characteristics

$$
F(t):=\sum_{n \geq 0} \chi\left(\operatorname{Hilb}_{n}^{(m)}\right) t^{n} \in \mathbb{Z}[[t]]
$$

Then the Donaldson-Thomas-type invariants $\mathrm{DT}_{n}^{(m)}$ are defined as rational numbers via the equality of power series

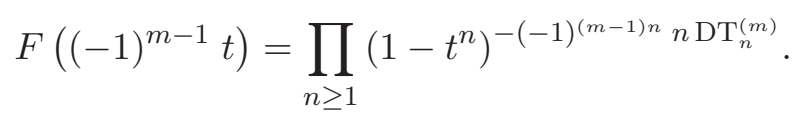

The shape of the above definition is motivated by Kontsevich and Soibelman [9]. Then, Reineke [17, Theorem 3.2] states that $\mathrm{DT}_{n}^{(m)} \in \mathbb{N}$ and provides a formula in terms of sums of binomial coefficients. This result is proven for a more general class of examples by Reineke in [16, Theorem 5.9 and formula (15)]. It is absolutely remarkable that the invariants $\mathrm{DT}_{n}^{(m)}$ are exactly the coefficients of the transformation matrix $C$ of Lemma 12 below:

$$
C_{s t}=\mathrm{DT}_{s / t}^{(t w-1)}
$$

\footnotetext{
${ }^{5}$ Namely, $\bar{M}_{0,0}(S, \beta)$ compactifies the moduli space of genus 0 curves in $S$ of class $\beta$.

${ }^{6}$ See $[11,12]$, where Maulik-Nekrasov-Okounkov-Pandharipande conjecture the precise relationship.
} 
Here $s, t \geq 1$ are such that $s / t \in \mathbb{N}$, and $w$ is as in Section 1.2. The matrix $C$ transforms the relative BPS state counts $n_{S}[d w]$ from Section 1.2 into the local BPS state counts $n_{d \beta}$ from Section 1.1. Albeit formula (1.7) provides a direct numerical connection, we are as of now not aware of a geometric connection as to why the transformation matrix from relative to local BPS state counts should be given by the invariants $\mathrm{DT}_{s / t}^{(t w-1)}$. Our proof of the integrality of the coefficients $C_{s t}$, via analysis of congruence relations of binomial coefficients, has much in common with the proof of $[17$, Theorem 3.2]. That theorem, however, is proven in the more general setting of [16]. Avoiding the extra formalism of [16], our proof is more direct.

\subsection{Outline}

The proof of Theorem 8 is split into two parts. In Section 2, Lemma 12 states the precise relationship between the local and relative BPS state counts that we consider. Each set of invariants is related to the other by means of an invertible matrix. In Section 3, we analyze congruence classes relating to the entries of this matrix. We prove that each entry is integer valued, which proves Theorem 8 .

\section{Combinatorics}

Let $S$ be a del Pezzo surface with smooth effective anticanonical divisor $E$ and let $\beta \in H_{2}(S, \mathbb{Z})$ be a non-zero effective primitive curve class. Consider two sequences of rational numbers

$$
\left\{N_{S}[d w]\right\}_{d \geq 1} \quad \text { and } \quad\left\{I_{K_{S}}(d \beta)\right\}_{d \geq 1}
$$

which we assume to be related, for all $d \geq 1$, by Equation (1.6). Define two sequences of rational numbers

$$
\left\{n_{S}[d w]\right\}_{d \geq 1} \text { and }\left\{n_{d \beta}\right\}_{d \geq 1}
$$

by means of Equations (1.2) and (1.4). Note that formula (1.4) is equivalent to the set of equations

$$
N_{S}[d w]=\sum_{k \mid d} \frac{1}{k^{2}}\left(\begin{array}{c}
k\left(\frac{d}{k} w-1\right)-1 \\
k-1
\end{array}\right) n_{S}[d w / k]
$$


Combining the formulas (1.1), (1.6) and (2.1) yields the following collection of formulas:

$$
\sum_{k \mid d} \frac{1}{k^{2}}\left(\begin{array}{c}
k\left(\frac{d}{k} w-1\right)-1 \\
k-1
\end{array}\right) n_{S}[d w / k]=(-1)^{d w+1} d w \sum_{k \mid d} \frac{1}{k^{3}} n_{d \beta / k}
$$

Fix a positive integer $N$ and, for $1 \leq d \leq N$, consider the formulas (2.2). In matrix form, this collection of formulas is expressed as

$$
R\left[n_{S}[d w]\right]_{d}=A \cdot L \cdot A^{-1}\left[(-1)^{d w+1} d w n_{d \beta}\right]_{d},
$$

where $R, A$ and $L$ are the following lower triangular $N \times N$ matrices:

$$
\begin{aligned}
R_{i j} & := \begin{cases}\frac{1}{(i / j)^{2}}\left(\begin{array}{c}
i / j(j w-1)-1 \\
i / j-1
\end{array}\right) & \text { if } j \mid i, \\
0 & \text { else, }\end{cases} \\
A_{i j} & :=(-1)^{i w+1} i w \delta_{i j}, \\
L_{i j} & := \begin{cases}\frac{1}{(i / j)^{3}} & \text { if } j \mid i, \\
0 & \text { else. }\end{cases}
\end{aligned}
$$

Note that $R$ is lower triangular and has determinant 1 .

Notation. For an integer $n$, denote by $\omega(n)$ the number of primes (not counting multiplicities) in the prime factorization of $n$. Moreover, let

$$
I(n):=\{k \in \mathbb{N}: k \mid n \text { and } n / k \text { is square-free }\} .
$$

Lemma 12. Define the $N \times N$ matrix $C$ as follows. If $t \mid s$, set

$$
C_{s t}:=\frac{(-1)^{s w}}{(s / t)^{2}} \sum_{k \in I(s / t)}(-1)^{\omega(s / k t)}(-1)^{k t w}\left(\begin{array}{c}
k(t w-1)-1 \\
k-1
\end{array}\right) .
$$

If $t \nmid s$, set $C_{s t}=0$. Then the sequences

$$
\left\{n_{S}[d w]\right\} \quad \text { and } \quad\left\{(-1)^{d w+1} d w n_{d \beta}\right\}
$$

for $1 \leq d \leq N$, are related via

$$
C \cdot\left[n_{S}[d w]\right]_{d}=\left[(-1)^{d w+1} d w n_{d \beta}\right]_{d}
$$


Moreover, $C$ has determinant 1 and is lower triangular. Thus, by Cramer's rule

$$
C \text { integral } \Longleftrightarrow C^{-1} \text { integral. }
$$

Proof. We start by writing $L=B \cdot \tilde{L} \cdot B^{-1}$, where

$$
\begin{aligned}
& \tilde{L}_{i j}= \begin{cases}1 & \text { if } j \mid i, \\
0 & \text { else, }\end{cases} \\
& B_{i j}=\frac{1}{i^{3}} \delta_{i j} .
\end{aligned}
$$

By Möbius inversion the inverse of $\tilde{L}$ is given by

$$
\left(\tilde{L}^{-1}\right)_{i j}= \begin{cases}(-1)^{\omega(i / j)} & \text { if } j \mid i \text { and } i / j \text { is square-free } \\ 0 & \text { else. }\end{cases}
$$

Then

$$
(A B)_{i j}=(-1)^{i w+1} \frac{w}{i^{2}} \delta_{i j}
$$

and

$$
\left((A B)^{-1}\right)_{i j}=(-1)^{i w+1} \frac{i^{2}}{w} \delta_{i j} .
$$

It follows from formula (2.3) that a matrix $C$ satisfying (2.5) is given by

$$
C=A L^{-1} A^{-1} \cdot R=A B \cdot \tilde{L}^{-1} \cdot(A B)^{-1} \cdot R .
$$

The matrix $C$ is lower triangular and has determinant 1 , as this is the case for both $A L^{-1} A^{-1}$ and $R$. A calculation then yields

$$
\left(A B \cdot \tilde{L}^{-1}\right)_{s r}= \begin{cases}(-1)^{s w+1} \frac{w}{s^{2}}(-1)^{\omega(s / r)} & \text { if } r \mid s \text { and } s / r \text { is square-free } \\ 0 & \text { else }\end{cases}
$$

and

$$
\left((A B)^{-1} \cdot R\right)_{r t}= \begin{cases}(-1)^{r w+1} \frac{r^{2}}{w} \frac{1}{(r / t)^{2}}\left(\begin{array}{cl}
r / t(t w-1)-1 \\
r / t-1
\end{array}\right) & \text { if } t \mid r, \\
0 & \text { else. }\end{cases}
$$

If $t$ does not divide $s$, then there is no integer $r$ such that $t|r| s$, so that $C_{s t}=0$. If, however, $t \mid s$, then

$$
C_{s t}=\frac{(-1)^{s w+1}}{(s / t)^{2}} \sum(-1)^{\omega(s / r)}(-1)^{r w+1}\left(\begin{array}{c}
r / t(t w-1)-1 \\
r / t-1
\end{array}\right),
$$


where the sum runs over all $r$ such that $t|r| s$ and such that $s / r$ is square free. Set $k=r / t$, so that, for $t$ dividing $s$

$$
C_{s t}=\frac{(-1)^{s w}}{(s / t)^{2}} \sum_{k \in I(s / t)}(-1)^{\omega(s / k t)}(-1)^{k t w}\left(\begin{array}{c}
k(t w-1)-1 \\
k-1
\end{array}\right)
$$

finishing the proof.

Lemma 12 reduces Theorem 8 to proving that the coefficients of the matrix $C$ are integers. This is achieved in Lemmas 15 and 16 of the next section.

\section{Integrality}

We start by stating the following lemma, which follows directly from the proof of Lemma A.1 of [15].

Lemma 13 (Peng). Let $a, b$ and $\alpha$ be positive integers and denote by $p$ a prime number. If $p=2$, assume furthermore that $\alpha \geq 2$. Then

$$
\left(\begin{array}{c}
p^{\alpha} a-1 \\
p^{\alpha} b-1
\end{array}\right) \equiv\left(\begin{array}{l}
p^{\alpha-1} a-1 \\
p^{\alpha-1} b-1
\end{array}\right) \quad \bmod \left(p^{2 \alpha}\right) .
$$

In the case that $p=2$ and $\alpha=1$, we have the following lemma:

Lemma 14. Let $k \geq 1$ be odd and let a be a positive integer. Then

$$
\left(\begin{array}{c}
2 k a-1 \\
2 k-1
\end{array}\right) \equiv(-1)^{a+1}\left(\begin{array}{c}
k a-1 \\
k-1
\end{array}\right) \bmod (4) .
$$

Proof. Note that

$$
\begin{aligned}
\left(\begin{array}{c}
2 k a-1 \\
2 k-1
\end{array}\right)= & \frac{2 k a-1}{2 k-1} \cdot \frac{2 k a-2}{2 k-2} \cdots \frac{2 k a-2 k+2}{2} \cdot \frac{2 k a-2 k+1}{1} \\
= & \frac{2 k a-1}{2 k-1} \cdot \frac{k a-1}{k-1} \cdots \frac{k a-k+1}{1} \cdot \frac{2 k a-2 k+1}{1} \\
= & \frac{(k a-1)(k a-2) \cdots(k a-k+1)}{(k-1)(k-2) \cdots 1} \\
& \cdot \frac{(2 k a-1)(2 k a-3) \cdots(2 k a-2 k+1)}{(2 k-1)(2 k-3) \cdots 1} \\
= & \left(\begin{array}{c}
k a-1 \\
k-1
\end{array}\right) \cdot \frac{(2 k a-1)(2 k a-3) \cdots(2 k a-2 k+1)}{(2 k-1)(2 k-3) \cdots 1}
\end{aligned}
$$


and hence

$$
\begin{aligned}
& \left(\begin{array}{c}
2 k a-1 \\
2 k-1
\end{array}\right)+(-1)^{a}\left(\begin{array}{c}
k a-1 \\
k-1
\end{array}\right) \\
& \quad=\left(\begin{array}{c}
k a-1 \\
k-1
\end{array}\right)\left((-1)^{a}+\frac{(2 k a-1)(2 k a-3) \cdots(2 k a-2 k+1)}{(2 k-1)(2 k-3) \cdots 1}\right) .
\end{aligned}
$$

It thus suffices to show that

$$
\frac{(2 k a-1)(2 k a-3) \cdots(2 k a-2 k+1)}{(2 k-1)(2 k-3) \cdots 1} \equiv(-1)^{a+1} \bmod (4) \text {. }
$$

Suppose first that $a$ is even, so that the left-hand side of (3.1) is congruent to

$$
\begin{aligned}
& \equiv \frac{(-1)(-3) \cdots(-(2 k-3))(-(2 k-1))}{1 \cdot 3 \cdots(2 k-3)(2 k-1)} \\
& \equiv(-1)^{k} \equiv(-1)^{a+1} \bmod (4),
\end{aligned}
$$

where the last congruence follows from the fact that $k$ is odd. Suppose now that $a$ is odd. Then the left-hand side of the expression (3.1) is congruent to

$$
\begin{aligned}
& \equiv \frac{2 k(a-1)+(2 k-1)}{2 k-1} \cdot \frac{2 k(a-1)+(2 k-3)}{2 k-3} \cdots \frac{2 k(a-1)+1}{1} \\
& \equiv 1 \equiv(-1)^{a+1} \bmod (4) .
\end{aligned}
$$

We return to the proof of Theorem 8. If $s=t$, then $C_{s t}=1$ is an integer, and we may thus henceforth assume that $t \mid s$, but $t \neq s$. Let $p$ be a prime number and $\alpha$ a positive integer. For an integer $n$, we use the notation

$$
p^{\alpha} \| n
$$

to mean that $p^{\alpha} \mid n$, but $p^{\alpha+1} \nmid n$. In order to prove that $C_{s t} \in \mathbb{Z}$, we show the following: if $p$ is a prime number such that

$$
p^{\alpha} \| \frac{s}{t}
$$

then

$$
p^{2 \alpha} \mid \sum_{k \in I(s / t)}(-1)^{\omega(s / k t)}(-1)^{k t w}\left(\begin{array}{c}
k(t w-1)-1 \\
k-1
\end{array}\right) .
$$


Fix a prime number $p$ and a positive integer $\alpha$ such that

$$
p^{\alpha} \| \frac{s}{t}
$$

We regroup the sum over $k \in I(s / t)$ as follows. Let $k \in I(s / t)$. For $s / k t$ to be square free, it is necessary that $p^{\alpha-1} \mid k$. This splits into the two cases

$$
p^{\alpha-1} \| k
$$

in which case $k / p^{\alpha-1} \in I\left(s / p^{\alpha} t\right)$ and

$$
p^{\alpha} \| k
$$

so that $k / p^{\alpha-1}=p l$ for $l \in I\left(s / p^{\alpha} t\right)$. Regrouping the terms of the sum (3.2) accordingly yields

$$
\sum_{l \in I\left(s / p^{\alpha} t\right)} \sum_{k \in\left\{p^{\alpha-1} l, p^{\alpha} l\right\}}(-1)^{\omega(s / k t)}(-1)^{k t w}\left(\begin{array}{c}
k(t w-1)-1 \\
k-1
\end{array}\right) .
$$

Thus, it suffices to show that for all $l \in I\left(s / p^{\alpha} t\right)$

$$
f(l):=\sum_{k \in\left\{p^{\alpha-1} l, p^{\alpha} l\right\}}(-1)^{\omega(s / k t)}(-1)^{k t w}\left(\begin{array}{c}
k(t w-1)-1 \\
k-1
\end{array}\right) \equiv 0 \bmod \left(p^{2 \alpha}\right)
$$

which we proceed to prove. There are two cases. In the above sum, either the sign $(-1)^{\omega(s / k t)}(-1)^{k t w}$ changes or it does not. The only case where the sign does not change is when $p=2, \alpha=1$, and both $t$ and $w$ are odd.

Lemma 15. Assume that either $p \neq 2$ or, if $p=2$, that $\alpha>1$. Then

$$
f(l) \equiv 0 \bmod \left(p^{2 \alpha}\right)
$$

Proof. In this case,

$$
\begin{aligned}
f(l) & = \pm\left(\left(\begin{array}{c}
p^{\alpha} l(t w-1)-1 \\
p^{\alpha} l-1
\end{array}\right)-\left(\begin{array}{c}
p^{\alpha-1} l(t w-1)-1 \\
p^{\alpha-1} l-1
\end{array}\right)\right) \\
& \equiv 0 \quad \bmod \left(p^{2 \alpha}\right)
\end{aligned}
$$

by Lemma 13 .

Lemma 16. Assume that $p=2$ and that $\alpha=1$. Then

$$
f(l) \equiv 0 \quad \bmod (4) .
$$


Proof. In this case,

$$
\begin{aligned}
f(l) & = \pm\left(\left(\begin{array}{c}
2 l(t w-1)-1 \\
2 l-1
\end{array}\right)+(-1)^{t w-1}\left(\begin{array}{c}
l(t w-1)-1 \\
l-1
\end{array}\right)\right) \\
& \equiv 0 \quad \bmod (4)
\end{aligned}
$$

follows from Lemma 14.

Therefore in both cases $f(l)$ is divisible by $p^{2 \alpha}$, and this finishes the proof that the entries of $C$ are integers. Consequently, by Lemma 12, the proof of Theorem 8 is complete.

\section{Acknowledgments}

M.v.G would like to thank T. Graber for introducing him to the subject of Gromov-Witten theory and for many enlightening discussions on curve counting that formed the basis for the present paper. M.v.G would like to extend special thanks to Y. Ruan, who has provided valuable guidance on the aspects of this paper relating to mirror symmetry. The authors would like to thank N. Yui, R. Abouaf and M. Florence for many helpful comments that improved the quality and readability of the paper. The authors would like to extend special thanks to the referees, whose reports greatly improved the quality of the present paper. Part of the research reported here was performed while the authors were students at the California Institute of Technology. This paper was completed while M.v.G was in residence at the Fields Institute for the thematic program: Calabi-Yau Varieties: Arithmetic, Geometry and Physics, July to December 2013. M.v.G would like to thank the Fields Institute for its hospitality and generous support. M.v.G. was supported by a Fields Postdoctoral Fellowship for the Fields major thematic program on Calabi-Yau Varieties: Arithmetic, Geometry and Physics from July to December 2013.

\section{References}

[1] J. Bryan and R. Pandharipande, BPS states of curves in Calabi-Yau 3-folds, Geom. Topol. 5 (2001), 287-318.

[2] T.-M. Chiang, A. Klemm, S.-T. Yau and E. Zaslow, Local mirror symmetry: calculations and interpretations, Adv. Theor. Math. Phys. 3(3) (1999), 495-565. 
[3] A. Gathmann, Relative Gromov-Witten invariants and the mirror formula, Math. Ann. 325 (2003), 393-412.

[4] M. Gross, R. Pandharipande and B. Siebert, The tropical vertex, Duke Math. J. 153(2) (2010), 297-362.

[5] R. Gopakumar and C. Vafa, M-theory and topological strings - I, arXiv:hep-th/9809187, 1998.

[6] R. Gopakumar and C. Vafa, M-theory and topological strings - II, arXiv:hep-th/9812127, 1998.

[7] D. Joyce and Y. Song, A theory of generalized Donaldson-Thomas invariants, Mem. Amer. Math. Soc. 1020 (2012), iv+199pp.

[8] Y. Konishi, Integrality of Gopakumar-Vafa invariants of toric CalabiYau threefolds, Publ. Res. Inst. Math. Sci. 42(2) (2006), 605-648.

[9] M. Kontsevich and Y. Soibelman, Stability structures, motivic Donaldson-Thomas invariants and cluster transformations, arXiv:0811.2435.

[10] Y. Manin, Generating functions in algebraic geometry and sums over trees, in 'The Moduli Space of Curves', eds R. Dijkgraaf, C. Faber and G. van der Geer (Texel Island, 1994), Progr. Math., 129, Birkhäuser, Boston, MA, 1995, 401-417.

[11] D. Maulik, N. Nekrasov, A. Okounkov and R. Pandharipande, GromovWitten theory and Donaldson-Thomas theory I, Compos. Math. 142 (2006), 1263-1285.

[12] D. Maulik, N. Nekrasov, A. Okounkov and R. Pandharipande, GromovWitten theory and Donaldson-Thomas theory II, Compos. Math. 142 (2006), 1286-1304.

[13] R. Pandharipande, Hodge integrals and degenerate contributions, Comm. Math. Phys. 208 (1999), 489-506.

[14] R. Pandharipande, Three questions in Gromov-Witten theory, Proceedings of the International Congress of Mathematicians (Beijing, 2002), II , Higher Education Press, 503-512.

[15] P. Peng, A simple proof of Gopakumar-Vafa conjecture forlocal toric Calabi-Yau manifolds, Comm. Math. Phys. 276 (2007), 551-569. 
Integrality of relative BPS state counts of toric del Pezzo surfaces 687

[16] M. Reineke, Cohomology of quiver moduli, functional equations, and integrality of Donaldson-Thomas type invariants, Compos. Math. 147(3) (2011), 943-964.

[17] M. Reineke, Degenerate cohomological Hall algebra and quantized Donaldson-Thomas invariants for m-loop quivers, Doc. Math. 17 (2012), 1-22.

[18] M. Reineke and T. Weist, Refined GW/Kronecker correspondence, Math. Ann. 355(1) (2013), 17-56.

[19] N. Takahashi, Log mirror symmetry and local mirror symmetry, Comm. Math. Phys. 220(2) (2001), 293-299.

KIAS, 85 HoEgIro

Dongdaemun-Gu, Seoul 130-722

REPUBLIC OF KOREA

E-mail address: vangarrel@kias.re.kr

Department of Mathematics

Kutztown University of Pennsylvania

15200 KutzTown RoAD

KutzTown

PA 19530

USA

E-mail address: wong@kutztown.edu

Department of Mathematics

California Institute of Technology

MC 253-37

1200 East CALifornia Boulevard

PASADENA

CA 91125

USA

E-mail address: gzaimi@caltech.edu

ReCEIVEd JANuARY 10, 2014 
